\title{
Predicting the effect of climate change on wildfire behavior and initial
}

\section{attack success}

\author{
Jeremy S. Fried
}

USDA Forest Service Pacific Northwest Research Station, Forest Inventory and Analysis

J. Keith Gilless (Corresponding Author)

University of California, Berkeley, Agricultural and Resource Economics

Berkeley, CA 94720-3100 (510)642-7171 gilless@nature.berkeley.edu

William J. Riley

Lawrence Berkeley National Laboratory Center for Isotope Geochemistry

Tadashi J. Moody

University of California, Berkeley Environmental Science, Policy, and Management Clara Simon de Blas

Universidad Rey Juan Carlos

Katharine Hayhoe

ATMOS Research and Consulting

Max Moritz

University of California, Berkeley Environmental Science, Policy, and Management Scott Stephens

University of California, Berkeley Environmental Science, Policy, and Management Margaret Torn

Lawrence Berkeley National Laboratory Center for Isotope Geochemistry 
Predicting the effect of climate change on wildfire behavior and initial attack success

\begin{abstract}
This study focused on how climate change-induced effects on weather will translate into changes in wildland fire severity and outcomes in California, particularly on the effectiveness of initial attack at limiting the number of fires that escape initial attack. The results indicate that subtle shifts in fire behavior of the sort that might be induced by the climate changes anticipated for the next century are of sufficient magnitude to generate an appreciable increase in the number of fires that escape initial attack. Such escapes are of considerable importance in wildland fire protection planning, given the high cost to society of a catastrophic escape like those experienced in recent decades in the Berkeley-Oakland, Santa Barbara, San Diego, or Los Angeles areas. However, at least for the three study areas considered, it would appear that relatively modest augmentations to existing firefighting resources might be sufficient to compensate for changeinduced changes in wildland fire outcomes.
\end{abstract}


Predicting the effect of climate change on wildfire behavior and initial attack success

\section{Introduction}

Previous analyses of wildfire and climate change have suggested that the area burned and number of fires that escape initial attack suppression would increase, sometimes dramatically, in northern California under a double-CO2 scenario (Fried et al. 2004; Torn and Fried 1992). In one extrapolation of simulation modeling results to all nonfederal lands in this region, a double-CO2 climate was predicted to lead to a doubling in the frequency of escaped fires (Fried et al. 2004). This prediction is sobering, because escaped fires have the potential to become very large, damaging fires. Many analysts have noted that given the importance of extreme fire weather in California, it is critical that we better understand how this weather is impacted by climate change (e.g., Davis and Michaelsen 1995). Prior studies of the impacts of climate change on wildfire have raised several important follow-on questions: Will climate change lead to changes in the number of fires (i.e., to fire occurrence), in fire rate-of-spread (ROS) distributions, or in the beginning and end dates of fire season for which fire agencies must be fully staffed? What additional firefighting resources would be needed to compensate for such changes? How much would reductions in greenhouse gas $(\mathrm{GHG})$ emissions significantly moderate the projected impacts? Although much more work remains to be done to address these questions completely, this preliminary analysis indicates that several existing models can be extended to provide valuable insights into the impact of climatic change on wildfire severity and outcomes.

Although the general approach employed in this study is similar to that used in Fried et al. (2004) and Torn and Fried (1992), the results reported here were derived using a more sophisticated stochastic model of initial attack on wildfires, and new methods of downscaling scenario data from global climate models (Cayan et al. 2006). The techniques employed are 
Predicting the effect of climate change on wildfire behavior and initial attack success

outlined in greater detail in Fried et al. (2006b), but are here applied to three administrative units of the California Department of Forestry and Fire Protection's (CDF) - the Amador-El Dorado (AEU), San Bernadino (BDU) and Santa Clara (SCU). Projections of the effects of climate change on wildfire for higher (business-as-usual) and reduced-anthropogenic-emission scenarios are presented below for each of these areas. 


\section{Methods}

This study relied on models and data drawn from California's strategic fire planning system to estimate aspects of climate change relevant to wildland fire at three levels of analysis. First, 150 years of simulated daily weather variables were generated for the period from 1950 to 2099 by downscaling from general circulation model simulations under two greenhouse gas (GHG) emissions scenarios. This data was divided into three periods: a baseline reference period from 1961 to 1999 denoted henceforth as BASE; a period in the middle of this century (2035 to 2064) denoted as MIDCEN; and a period at the end of the century (2070 to 2099) denoted as ENDCEN. Second, these daily weather data were used to estimate 2 p.m. wildfire ROS and burning index (BI) values using the Fire Behavior Dispatch Modeling System (FBDMOD) (CDF 1992), a program patterned after the National Fire Danger Rating System (Deeming et al. 1977). Estimates of these key wildfire behavior variables were made for a wide range of fuel and slope conditions in each of the three study areas. Finally, the impact of these predicted changes in wildfire behavior on initial attack containment efforts were assessed using the California Fire Economics Simulator version 2 (CFES2) (Fried and Gilless 1999), a stochastic computer model developed for the California Department of Forestry and Fire's (CDF) fire protection planning program. Detailed methods and assumptions for each level of this analysis are outlined below.

\subsection{The Study Areas}

The CDF's Amador-El Dorado Unit encompasses the portions of Amador and El Dorado counties where the CDF has primary responsibility for wildfire suppression, with assistance from cooperating local and federal agencies. The San Bernadino Unit encompasses similarly defined 
Predicting the effect of climate change on wildfire behavior and initial attack success

portions of San Bernadino, Inyo, and Mono counties; the Santa Clara Unit portions of Santa Clara, Stanislaus, Alameda, Contra Costa, and San Joaquin counties. These three areas were selected because they span a wide environmental and social gradient, from the foothills of the Sierra Nevada, to the coastal San Francisco Bay area, to interior areas of Southern California.

The CDF (and the CFES2 model) stratifies administrative units into fire management analysis zones (FMAZs) defined by fuel type (e.g., grass, brush — an indicator of the fire regime) and population density (low, medium, or high - an indicator of the extent to which issues of wildland urban interface are germane). The fuel models used in the FMAZ stratification of AEU (A, B, C, F, H, and U), BDU (A, B, F, T), and SCU (A, B, F, G) (Table 1) are taken from the National Fire Danger Rating System (Deeming et al., 1977).

CFES2 simulates initial attack on wildland fires at a number of representative fire locations (RFLs) within each FMAZ. RFLs are chosen on the basis of historical fire locations, and are characterized by a fuel model, slope class, herbaceous vegetation type, climate class, and representative fire weather station - a set of attributes collectively referred to as a "fuel combination."

The fire weather stations utilized in this study for AEU were located at Bald Mountain (lat 3854 3, long 12041 8) and Georgetown (lat 3855 10, long 12054 0); for BDU at Saugus (lat 3425 30, long 11831 30), West Riverside (lat 340 35, long 11727 0), Devore (34 035 , long 11727 0), and Apple Valley (lat 3436 0, logn 11710 0); and for SCU at Sunol (lat: 3733 11, long 12150 39), Del Puerto (lat 37 0 48, long 121 7 30) and Morgan Hill (lat 37 6 39, long $1213812)$. 


\subsection{Climate Forcing}

Simulated localized daily fire weather variables were derived for the BASE, MIDCEN and ENDCEN periods from simulations made using two atmosphere-ocean general circulation models (AOGCMs): the NOAA-GFDL CM2.1 (Delworth et al. 2005), denoted henceforth as GFDL; and the DOE-NCAR Parallel Climate Model (Washington et al. 2000), denoted as PCM. Simulations are forced by the IPCC Special Report on Emission Scenarios mid-high (A2) and lower (B1) emissions scenarios (SRES, Nakićenović et al. 2000) described in greater detail in Cayan et al. (2006).

The GFDL and PCM monthly temperature and precipitation fields for the A2 and B1 scenarios were statistically downscaled to daily values for areas with a resolution of $1 / 8^{\circ}$, or about 12 kilometers (km) (7 miles) (Wood et al. 2002), for each fire weather station using a technique that maps the probability density functions for modeled monthly and daily precipitation and temperature for the BASE period onto those of gridded historical observed data, so the mean and variability of both monthly and daily observations are reproduced. Daily average relative humidity values that correspond to the daily temperature and precipitation for each grid cell were generated using the Variable Infiltration Capacity (VIC) distributed land surface hydrology model. The bias correction and spatial disaggregation technique employed was originally developed for adjusting AOGCM output for long-range streamflow forecasting (Wood et al. 2002), and later adapted for use in studies examining the hydrologic impacts of climate change (VanRheenen et al. 2004).

In addition to daily maximum temperature, minimum temperature, precipitation, and humidity, historical weather data for each weather station identified above was used in 
Predicting the effect of climate change on wildfire behavior and initial attack success

conjunction with the modeled variables to project several key inputs for the FBDMOD fire behavior prediction software - daily 2 p.m. temperature and relative humidity, precipitation duration, maximum and minimum relative humidity, 10-hour fuel moisture, wind direction and speed, and state of the weather for all periods. A more detailed explanation of the methods used to make these projections can be found in Fried et al. (2006b).

\subsection{Fire Behavior}

Daily 2 p.m. fire rate of spread and burning index (a proxy for intensity) was predicted using FBDMOD for the three periods (BASE, MIDCEN, and ENDCEN) for each fuel combination in AEU, BDU, and SCU under the higher (A2) and lower (B1) emissions scenarios as simulated by the GFDL and PCM models. Weather station descriptive information required by FBDMOD (elevation, latitude, date of vegetation green-up, date of first killing frost) was obtained from the fire weather archives of the National Fire and Aviation Management Web (http://famweb.nwcg.gov/weatherfirecd/index.htm). These predictions describe how fires would spread at near daily worst-case rates, without crowning or spotting, through continuous fuels on a uniform slope (NWCG 2002). Downward adjustments are made to these rates in the CFES2 model to reflect the diurnal patterns of fire behavior (Gilless and Fried 1999).

Daily-resolved fire behavior predictions were divided into five inter-annual seasons for CFES2 analysis: low 1 (January 1 to May 15), transition 1 (May 15 to June 15), high (June 15 to October 15), transition 2 (October 15 to November 15) and low 2 (November 15 to December 31). These same seasons are used in CFES2 to characterize the availability of fire suppression resources (Fried and Gilless 1999). We were forced to hold these dates constant for this study 
Predicting the effect of climate change on wildfire behavior and initial attack success

given the time constraints of this study, although clearly the effects of climate change on the seasonal timing and effect of the high fire season and institutional response to such changes is obviously an important area for further research.

Distributions of ROS (and BI) for a particular season are consistently bimodal with a narrow distribution characterized by a local (and sometimes global) peak at a low rate of spread coupled with a distinctly separate, unimodal distribution covering a wider (and faster) range of spread rates. Each compound seasonal distribution for these variables was therefore split into two parts, with a simple constant rate used to characterize the values for slow-moving fires, and a beta distribution used to characterize more rapidly-moving fires (Gilless and Fried 1999). Determining both where to split the data and the best fitting beta curve for faster moving fires was done by iterative use of a chi-square test and the moments method of curve fitting.

\subsection{Initial Attack Simulation}

CFES2's event-based simulation framework is based on stochastic modules describing fire occurrence, fire behavior, and the effectiveness of firefighting resources in constructing fireline (Gilless and Fried 1999a, 1999b; Fried and Gilless 1988), and local information on the availability of suppression resources and their response times to different RFLs. CFES2 simulates fire events with specific locations and start dates and times, and estimates the behavior (rate of spread or burning index) of these fires by drawing from distributions of potential fire behavior (ROS and BI) for the appropriate fuel combination and season.

CFES2 was used to generate estimates of the frequency of escaped fires and the area burned by contained fires for the BASE, MIDCEN, and ENDCEN periods, for the A2 and B1 
Predicting the effect of climate change on wildfire behavior and initial attack success

scenarios, based on estimated distributions for fire ROS and BI, for both the GFDL and PCM AOGCMs. For each combination of period, GHG emission scenario, and AOGCM, 200 years of simulated results were obtained to allow for statistical characterization of the stochastic outcomes.

When using CFES2 to conduct comparative analysis, any of a number of kinds of results can prove interesting, including changes in the frequency of escapes, area burned in contained fires by season, size class, and geographical unit; percentage of fires that are successfully contained within predetermined size limits; and even utilization frequency and dispatching costs of any particular firefighting resource or group of resources. And, because CFES2 is a stochastic simulator that produces as many potential realizations as desired, we can look beyond a single, deterministic result for any attribute of interest, and report information on variability (e.g., standard errors) along with expected values, or even selected percentiles of the distribution of realizations (Fried et al. 2006a). This analysis focused on estimates of the expected value (and standard errors) of escaped fires per year by FMAZ.

Important assumptions were made in our CFES2 simulations to make the analysis tractable, and these assumptions obviously temper the definitiveness of the results presented below - many parameters that may well change over the next century were held constant, including fire occurrence, fuel models, the amount, positioning, and productivity of firefighting resources, and population density. 
Predicting the effect of climate change on wildfire behavior and initial attack success

3. Results

3.1. Climate

The impact of global climate change on daily weather characteristics at the location of the different fire weather stations appeared to differ markedly by variable. In some cases the projected changes are larger under a higher emissions scenario (A2 versus B1), illustrating the sensitivity of fire weather to emissions pathways and the degree of global change; in others, the GFDL model shows changes that are distinctly different from the PCM model, highlighting inter-model uncertainty; and in some cases, changes are not consistent either between models or scenarios, displaying a more random component that may be indicative of the sensitivity of that variable to multiple and interactive changes in and feedbacks between climate characteristics.

Projected changes in 2 p.m. temperature appeared relatively robust, consistent across both models and scenarios. Projections of relative humidity $(\mathrm{RH})$ displayed a strong dependence on both the model and the emissions scenario, with GFDL-based projections being consistently drier than PCM-based projections, and the magnitude of the projected changes over time being greater for the A2 scenario. The inter-model differences were more pronounced when considering only low-humidity days (defined as 2 p.m. RH less than 15\%).

Precipitation is similar to $\mathrm{RH}$ in that the sign of the change is determined by the model, but the magnitude is a function of the emissions scenario (larger changes for A2 relative to B1 for each model).

Consistent with being parameterized as proportional to relative humidity and 
precipitation and inversely proportional to temperature, we did not see consistent changes in projected 10-hr FM values. Changes in wind speed were extremely inconsistent, displaying no uniform dependence on either model or scenario. The observed changes between periods were particularly significant, likely reflecting the fact that wind speed is parameterized as such a complex function of daily weather characteristics and wind direction for each location that the multiple changes in temperature, humidity, wind direction, and other factors already observed are interacting here to simultaneously increase and decrease wind speed, producing little net change. It is also likely a result of the fact that this approach does not take into account modelsimulated changes in the wind-related characteristics of the daily weather systems. A comparison of these wind direction and speed projections with high-resolution regional model-generated values is planned for the future, to evaluate the ability of this approach to capture projected shifts in wind owing to climate change.

\subsection{Fire Behavior}

Changes in fire behavior predictions between periods were analyzed using Tukey's Honestly Significant Difference (HSD) test for multiple comparisons, a method based on the range of the sample means that does not mandate assumptions of normality or equal variances, and which is conservative when comparing samples of unequal size. Neither students-t test nor analysis of variance (ANOVA) would have been suitable for this data, because the values of ROS and BI for a given period are not distributed normally, and simple comparisons using t-tests would inflate the probability of declaring a significant difference between periods. Comparisons 
Predicting the effect of climate change on wildfire behavior and initial attack success

were made both for mean ROS for the portions of the ROS distributions modeled as beta distribution (Gilless and Fried 1999 and for the highest 5\% of the values in the entire ROS distribution (i.e., for the portion corresponding to the most "extreme" fire behavior).

Table 2 shows mean rates of spread (ROS) for the high fire season (June 15 to October 15) under GFDL scenario A2 for the 28 fuel-weather station-slope class-period combinations utilized in this study. Table 3 shows similar results for GFDL scenario B1. Although the number of significant differences in mean ROS is similar for the two scenarios between the BASE period and the MIDCEN period, the regional pattern of these increases is not consistent. The number of significant differences differs dramatically, however, when the BASE period is compared to the ENDCEN period (21 significant differences for A2 vs. only 8 for B1), indicating the divergence between the two scenarios is greater over time. Similar tables for the PCM scenarios show far fewer significant differences, although they are consistent with the GDFL scenarios in showing a greater rightward shift in the ROS and BI distributions for the A2 scenario than for the B1 scenario. Similarly, comparisons based only on the $95^{\text {th }}$-plus percentile values showed a greater evidence of rightward shifts in these distributions for the A2 scenario than for the B1 scenario for both the GDFL- an PCM-based simulations, although the number of significant differences was much smaller, and largely limited to shrub fuel models.

\subsection{Initial Attack Simulation}

The two key outcomes of initial attack on wildfire that are predicted by the CFES2 model are the expected number of ESL fires (those that would exceed simulation limits on time to 
Predicting the effect of climate change on wildfire behavior and initial attack success

containment or size upon containment) and the expected area burned for all fires contained within those same limits. The ESL fires can be interpreted loosely as fires that would "escape" initial attack, because the time and size limits used to define them are primarily a reflection of what policymakers regard as an escape. In some cases, although probably not for the three administrative units for which these results were generated, these time and size limits might also be used to express bounds on what is considered a range within which the simulation process is valid (i.e., one within which topography or firefighter fatigue does not fundamentally change the nature of what is being simulated, or imply that a fire would have moved into an "extended attack" phase in which additional resources would be dispatched or firefighting tactics would need to be adjusted). Past analyses using the CFES2 model have indicated that the model is most useful when the focus is placed on comparing changes in the number of ESLs between scenarios, i.e., when it is used for marginal analysis, rather than on making comparisons to historical averages of "escapes" (Fried et al. 2006a). The reasons for this include sensitivity of the historical number of ESLs to changes in the availability of firefighting resources, their stationing, and the dispatch policies governing their use, as well as changes in the policy goals that are used to define escapes in terms of time to fire containment or size at time of containment.

Tables 4 and 5 show the mean annual differences in the number of ESLs among periods, by administrative unit, under the GDFL A2 and B1 scenarios, respectively. The predicted distribution of the ESL fires by fuel model was quite close to the historical record, leading us to conclude that BASE simulation results provided a good basis for both relative and absolute comparisons with the predicted wildfire outcomes for MIDCEN and ENDCEN. A clear signal emerges from this data, with $\sim 2-5$ additional ESLs being predicted per year for each 
Predicting the effect of climate change on wildfire behavior and initial attack success

administrative unit the A2 scenario, compared to $~ 1-3$ for the B1 scenario. There are a few significant decreases in the mean number of ESLs per year moving from the MIDCEN to ENCEN periods, but all other significant changes show the expected sign. Results are not presented for the future fire weather predicted using the PCM model, but given the smaller shifts in the distribution of ROS predicted using that model, the results would predictably be more modest that those derived using the GDFL model.

In an effort to assess what additional resources would be to offset the predicted changes in fire outcomes, several what-if simulations were run for the AEU administrative unit in which a few "new" fire engines were added to the preexisting configuration of firefighting resources for the ENDCEN period, to be nominally dispatched from the fire stations generally providing the first-responding engines to simulated wildland fires. This augmentation of resources was generally sufficient to reduce the frequency of ESLs to that projected for the BASE period. 


\section{Conclusions}

As climate change and population growth unfold over the coming century, we can expect changes in the wildland fire system to manifest themselves through several pathways. Climateinduced changes in weather will directly affect the behavior of vegetation fires by altering their rate of spread or intensity in ways that ultimately affect their outcomes: burned area, damage to natural resources or infrastructure, fire agency budgets and suppression expenditures, and number of fires that escape initial attack and therefore have the potential to become larger or very costly.

Just as importantly, but much more difficult to forecast, we can expect climate change to alter vegetation composition, conceivably to an extent that leads to substantial changes in the fuels available to burn, thereby affecting fire behavior. For example, if timber species die out and are replaced by shrubs, there might be less total burnable fuels and fewer commercial natural resources at risk, but fire rates of spread would likely increase. If additional moisture results in conversion from shrub or grassland vegetation to forests, on the other hand, the converse might occur.

Apart from climate change, we know that population growth will almost certainly result in additional area covered in wildland vegetation becoming incorporated into California's extensive wildland-urban interface. As the wildland-urban interface spreads, the values at risk in this type of development will inexorably result in public demands for greater expenditures on firefighting resources, and for more aggressive initial attack to protect the increased values at risk. To the extent that these demands are met simply by increased use of existing resources (e.g., 
Predicting the effect of climate change on wildfire behavior and initial attack success

dispatch five engines to a fire instead of two), then this would act as a countervailing force tending to reduce any climate change-induced increase in the frequency of escaped fires without fundamentally changing the fixed costs of fire control. The effects of infrastructure development in the wildland-urban interface on the marginal costs of fire control are difficult to generalize, given their site and situation specificity, and the likelihood that wildland-urban interface communities will actively pursue options to reduce their hazard exposure in ways that complement the activities of state and federal fire agencies.

Growth in the wildland-urban interface (WUI) in recent years has placed more people in contact with wildlands, thereby increasing the likelihood of human-caused ignitions. In California, the area in this condition has grown by $9 \%$ in a decade (Hammer et al. in press). While there are as yet no signs of this trend abating in California, remarkable reductions in the rate of conversions from wildland to residential have been demonstrated in Oregon as a result of land use controls imposed in 1980 (Lettman et al. 2004).

This white paper focuses primarily on the first pathway noted above- how climate change-induced effects on weather will translate into changes in wildland fire severity and outcomes, particularly on the effectiveness of initial attack at limiting the number of fires that escape initial attack. The other pathways are not less important, but could not be addressed within the severe time, personnel, and resource limitations under which this work was undertaken.

Prior research on this issue indicated that there is a potential for significant increases in the number of fires escaping initial attack, particularly in areas in which the fuel matrix is 
Predicting the effect of climate change on wildfire behavior and initial attack success

dominated by grass and brush. These results were driven primarily by predicted increases in wind speeds which were used to directly adjust wind speeds associated with modeled, representative fires. Those findings, however, were derived for a single climate change scenario, very coarse-scale AOGCMs, and a rather simplistic deterministic simulation model of initial attack on wildland fire (CFES-IAM).

In contrast, the analysis reported here used two state-of-the-art AOGCMs (GFDL and PCM), new downscaling techniques to link the outputs of those AOGCMs to historical data from local weather stations, and a much more sophisticated stochastic simulation model of initial attack on wildland fire (CFES2) that was developed specifically to address the deficiencies noted in the model used in the prior research on this topic. Using this more rigorous approach with data from three CDF administrative units, this study's primary findings can be briefly summarized as follows.

First, the subtle shifts in fire behavior of the sort that might be induced by the climate changes anticipated for the next century are of sufficient magnitude to generate an appreciable increase in the number of fires that escape initial attack. It is important to remember that even a few additional ESLs per decade [would] could have significant public policy implications, given the high cost to society of a catastrophic escape like those experienced in recent decades in the Berkeley-Oakland, Santa Barbara, San Diego, or Los Angeles areas.

Second, comparison of the higher A2 and lower B1 emissions scenarios shows that the lower emissions scenario seems to be sufficient to produce modest reductions in the anticipated negative impacts on wildland fire severity and outcomes relative to the higher A2 scenario.

Third, this analysis is sensitive to the choice of AOGCM. Projections of certain climate 18 
Predicting the effect of climate change on wildfire behavior and initial attack success

variables that display strong relationships to fire conditions and spread (e.g., wind speed, 10-hr fuel moisture) were more sensitive to the model than the emissions scenario. Carrying these projections through to simulations of wildland fire severity, we found the PCM-generated climate scenarios (which were in general wetter than and not as warm as GFDL) to result in more modest predicted changes in wildland fire severity and outcomes than GFDL-generated climate-change scenarios.

Fourth, the magnitude of the climate change-induced changes in wildland fire severity and outcomes was less than reported in prior work, and we suspect that this is primarily owing to different assumptions with respect to how wind speed is treated in the process of downscaling AOGCM climate simulations. The method used in this study was more conservative, sampling from historical distributions, but, lacking input from larger-scale dynamics as represented by a regional climate model, it may underestimate the effect of climate change on wind fields. Further work on how to combine the historical range of wind speeds observed at weather stations with dynamic simulations of changes in regional to local-scale wind fields under climate change scenarios and evaluation of their overall impact on wildfire severity and spread will be a high priority for the research team who collaborated to produce this report.

Fifth, we conclude that the effect of climate change on wildland fire outcomes might be compensated for with a modest augmentation to existing resources. A modest augmentation of firefighting resources in all CDF administrative units and counties which provide wildland fire protection services for the State under contract, however, might translate at the statewide level into a significant budget augmentation. 
Predicting the effect of climate change on wildfire behavior and initial attack success

Sixth, although the existing "fire season" during which the CDF maintains a fully staffed organization is more a reflection of annual fire occurrence patterns than anticipated fire behavior, the results of climate change on fire behavior predicted by using the methods employed in this study suggest that fire behavior might play a more significant role in determining the length of the fire season in the future. Further exploration of this possibility will, of course, need to be coordinated with work on how fire occurrence patterns might change as a result of both climate and demographic changes over the next century.

Seventh, it might be useful for future work to attempt to factor in vegetation change resulting from climate change, possibly by using the newer 40 -fuel model system in BEHAVE 2, or perhaps the 256-fuel model matrix of the fuel characteristics classification system.

Eighth, although this study has focused on a consideration of the impact of climate change on rate of spread, it may be just as important to consider the impacts of climate change and vegetation management activities on the potential for crown fires. Some of the models currently in use for assessing crown fire potential would have benefit for extension of this research of being linkable to the Forest Inventory Analysis (FIA) data on forest condition collected by the USDA Forest Service (USFS).

Finally, in contrast to prior work, the use in this study of a stochastic model of initial attack demonstrates the value of being able to generate standard errors on the mean values of predicted outcomes for hypothesis testing, as well as for characterizing the impact of climate change on the extreme values of fire ROS distributions.

Extending our analysis to cover the public forests managed by the USFS on the north 
Predicting the effect of climate change on wildfire behavior and initial attack success

coast or in the upper elevations of the Sierra Nevada would be difficult owing to differences between the USFS Fire Program Analysis (FPA) model and the California Department of Forestry and Fire Protection's CFES2 model for analysis of initial attack on wildland fires. The two models are similar in many ways, but the FPA model does not incorporate a stochastic treatment of key simulation elements (e.g., fire occurrence or rate of spread), and therefore its simulation outputs cannot be analyzed by using the same significance-testing methods. The same methods could, however, be used to downscale GCM climate projections for a similar analysis of the impact of climate change on fire rates of spread, and a deterministic analysis could be performed by using the FPA model of the effects of those changes. It is also likely that a more sophisticated analysis of the impact of climate change on wildfire on public and private lands in California would entail the development of separate prediction models of the effects on fire occurrence, as most fires on CDF-protected lands are of anthropogenic origin; whereas lightningcaused fires constitute a higher proportion of fire ignitions on much of the land protected by the USFS.

We believe that the research summarized in this paper will prove to be at least as valuable as any attempt would have been to estimate statewide impacts, if not more so, because of the problems and opportunities it has identified in our capacity to address the questions that motivated the study. In particular, it is now clear to the fire research community at the University of California, Berkeley, and to their network of collaborators at other institutions, that much more work is needed to validate some of our modeling approaches, or develop entirely new ones, to many of the elements of the system we are modeling. 
Predicting the effect of climate change on wildfire behavior and initial attack success

\section{Acknowledgment}

This work was partially supported by the Director, Office of Science, Office of Biological and Environmental Research, Climate Change Research Division, of the U.S. Department of Energy under Contract No. DE-AC02-05CH11231. 
Predicting the effect of climate change on wildfire behavior and initial attack success

\section{References}

California Department of Forestry and Fire Protection (CDF). 1992. FBDMOD: Fire behavior dispatch modeling system. Sacramento, California.

Cayan, D., Luers, A.L., Hanemann, M., Franco, G., and B. Croes. 2006. Scenarios of climate change in California: An Overview (In review for same issue of Climatic Change)

Davis, F. W., and J. Michaelsen. 1995. Sensitivity of fire regime in chaparral ecosystems to global climate change. In: Moreno, J.M., Oechel, W.C. (Eds) Global Change and Mediterranean-Type Ecosystems. Springer-Verlag, New York, 435-456.

Deeming, J. E., R. E. Burgan, and J. D. Cohen. 1977. The National Fire Danger Rating System 1978, General Technical Report INT-39, USDA Forest Service Intermountain Forest and Range Experiment Station, 63 pp.

Delworth, T. L., A. J. Broccoli, A. Rosati, R. J. Stouffer, et al. 2005. “GFDL's CM2 global coupled climate models-Part 1-Formulation and simulation characteristics.” J. Clim., in press.

Fried, J. S., and J. K. Gilless. 1988. Stochastic Representation of Fire Occurrence in a Wildland Fire Protection Model for California. Forest Science 34:948-955.

Fried, J. S., and J. K. Gilless. 1999. CFES2: The California Fire Economics Simulator Version 2 User's Guide, University of California, Division of Agriculture and Natural Resources Publication 21580, 92 pp.

Fried, J. S., J. K. Gilless, and J. Spero. 2006a. "Analysing initial attack on wildland fires using stochastic simulation.” International Journal of Wildland Fire 15:137-146. 
Predicting the effect of climate change on wildfire behavior and initial attack success

Fried, J.S., Gilless, J.K., Riley, W.J., Moody, T.J., Simon de Blas, C., Hayhoe, K., Moritz, M., Stephens, S., and M. Torn. 2006b. Predicting the effect of climate change on wildfire severity and outcomes in California: Preliminary analysis. California Climate Change Center White Paper CEC-500-2005-196-SF.

Fried, J. S., M. S. Torn, and E. Mills. 2004. "The impact of climate change on wildfire severity: A regional forecast for Northern California. Climatic Change 64:169-191.

Gilless, J. K., and J. S. Fried. 1999. "Stochastic representation of Fire Behavior in a Wildland Fire Protection Planning Model for California.” Forest Science 45:492-499.

Hammer, R.B., V.C. Radeloff, J.S. Fried and S.I. Stewart. [in press]. Wildland-urban interface growth during the 1990s in California, Oregon, and Washington. International J. of Wildland Fire.

Lettman, G.J., Azuma, D.L., Birch, K.R., Herstrom, A.A., Kline, J.D. 2004. Land use change on non-federal land in eastern Oregon, 1975-2001. Salem, OR: Oregon Department of Forestry. 42 p.

Nakićenović, N., J. Alcamo, G. Davis, B. de Vries, J. Fenhann, S. Gaffin, K. Gregory, A. Grübler,

T. Y. Jung, T. Kram, et al. 2000. IPCC Special Report on Emissions Scenarios. Cambridge University Press, Cambridge, United Kingdom and New York, New York.

National Wildfire Coordinating Group (NWCG). 2002. Gaining and Understanding of the National Fire Danger Rating System. Publication PMS 932, NFES 2665.

Torn, M. S., and J. S. Fried. 1992. "Predicting the impacts of global warming on wildland fire." Climatic Change 21(3):257-274. 
Predicting the effect of climate change on wildfire behavior and initial attack success

Van Rheenen, N. T., A. W. Wood, R. N. Palmer, and D. P. Lettenmaier. 2004. "Potential implications of PCM climate change scenarios for California hydrology and water resources." Climatic Change 62:257-281.

Washington, W. M., J. W. Weatherly, G. A. Meehl, A. J. Semtner, T. W. Bettge, A. P. Craig,

W. G. Strand, J. Arblaster, V. B. Wayland, R. James, and Y. Zhang. 2000. "Parallel climate model (PCM) control and transient simulations.” Climate Dynamics 16:755-774.

Wood, A. W., L. R. Leung, V. Sridhar, and D. P. Lettenmaier. 2004. "Hydrologic implications of dynamical and statistical approaches to downscaling climate model outputs." Climatic Change 62:189-216.

Wood, A. W., E. P. Maurer, A. Kumar, and D. P. Lettenmaier. 2002. "Long range experimental hydrologic forecasting for the eastern U.S.” J. Geophys. Res. 107(D20), 4429. 